\title{
新型吡唑酰基腿类化合物的设计合成、杀菌活性与分子对接研究
}

\author{
孙娜波 ${ }^{a}$ 沈钟华 ${ }^{b}$ 翟志文 ${ }^{b}$ 武宏科 ${ }^{b}$ \\ 翁建全 ${ }^{b}$ 谭成侠 $b$ 刘幸海 ${ }^{*}, b$

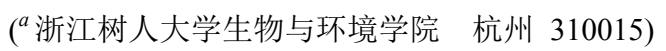 \\ ( ${ }^{b}$ 浙江工业大学化学工程学院 杭州 310014)
}

\begin{abstract}
摘要 以乙酰乙酸乙酯、原甲酸三乙酯、甲基肼等为原料，通过多步反应制备了一系列含吡唑的酰基䐂类化合物. 产 物结构均经过 ${ }^{1} \mathrm{H}$ NMR 和 HRMS 确证; 对所有化合物进行了杀菌活性测试, 结果表明部分化合物对花生褐斑病菌、苹 果轮纹病菌、水稻纹枯病菌、油菜菌核病菌具有较好的抑制效果, 同时将高活性化合物 $7 \mathbf{i}$ 与琥珀酸脱氢酶进行了对接, 计算结果显示化合物 7i 能与琥珀酸脱氢酶形成稳定的复合物, 化合物中的酰胺键能与酶中 Ser39 残基形成氢键作用. 关键词 吡唑; 酰基脲; 合成; 杀菌活性; 分子对接
\end{abstract}

\section{Design, Synthesis, Fungicidal Activity and Docking Study of Acyl Urea Derivatives Containing Pyrazole Moiety}

\author{
Sun, Nabo ${ }^{a} \quad$ Shen, Zhonghua ${ }^{b} \quad$ Zhai, Zhiwen $^{b} \quad$ Wu, Hongke ${ }^{b}$ \\ Weng, Jianquan $^{b} \quad$ Tan, Chengxia $^{b} \quad$ Liu, Xinghai ${ }^{*}, b$ \\ $\left({ }^{a}\right.$ College of Biology and Environmental Engineering, Zhejiang Shuren University, Hangzhou 310015) \\ ( ${ }^{b}$ College of Chemical Engineering, Zhejiang University of Technology, Hangzhou 310014)
}

\begin{abstract}
A series of novel acyl urea derivatives containing pyrazole moiety were synthesized from ethyl acetoacetate, triethyl orthoformate, methylhydrazine by multi-step reactions. Their structures were characterized by ${ }^{1} \mathrm{H}$ NMR and HRMS spectra. The target compounds were evaluated for their fungicidal activity. The results indicated that some of the title compounds displayed certain fungicidal activities against Cercospora arachidicola, Sclerotinia sclerotiorum, Rhizoctonia solani and Physalopora piricola. The docking results indicated the hydrogen bond formed between the amide group and Ser 39 residue.
\end{abstract}

Keywords pyrazole; acyl urea; synthesis; fungicidal activity; docking

琥珀酸脱氢酶抑制剂作用于病原菌线粒体呼吸电 子传递链上的蛋白复合体 II, 即琥珀酸脱氢酶或琥珀 酸-泛醌还原酶, 是被杀菌剂抗性行动委员会(FRAC)新 划分出来的一类作用机制和抗性机理相似的化合物 ${ }^{[1]}$. 自从靶向琥珀酸脱氢酶的莠锈灵(carboxin)和氧化莠锈 灵(oxycarboxin)问世后, 这类抑制剂已成为生产上一类 非常重要的杀菌剂品种, 同时新颖结构的该类杀菌剂不 断问世 ${ }^{[2]}$. 如近年来开发的联苯吡菌胺、氟唑环菌胺、 氟唑菌酰胺、吡唑萗菌胺、吡噻菌胺、呋吡菌胺、苯并 烯氟菌唑等. 该类杀菌剂分子结构变化比较大，且近年 来发现的大多含吡唑环 ${ }^{\left[{ }^{2}\right.}$ 5]. 另一方面, 嫝类化合物因

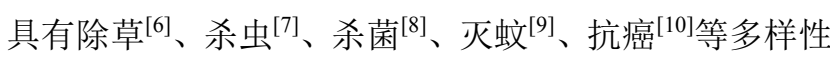
的生物活性而受到广泛关注. 一些含酰基脲类化合物已 经开发成功许多商品化的农药和医药. 本课题组在商品 化琥珀酸脱氢酶抑制剂和前期工作 ${ }^{[11 ~ 18]}$ 的基础上，根 据活性亚结构拼接、生物等排等方法, 将腿结构引入到 吡唑酰胺类化合物，同时考虑到先导化合物吡唑酰胺类 化合物大部分含氟, 尽可能在苯环上引入氟原子, 因此 合成了 12 个未见报道的吡唑酰基腿类新化合物 $7 \mathbf{a} \sim 71$, 设计思路如图 1 所示, 合成路线如 Scheme 1 所示.

\footnotetext{
* Corresponding author. E-mail: xhliu@zjut.edu.cn

Received February 3, 2017; revised March 30, 2017; published online April 18, 2017. LY16C14007)

国家自然科学基金(No. 31401691)和浙江省自然科学基金(No. LY16C14007)资助项目.
}

Project supported by the National Natural Science Foundation of China (No. 31401691) and the Zhejiang Provincial Natural Science Foundation of China (No. 


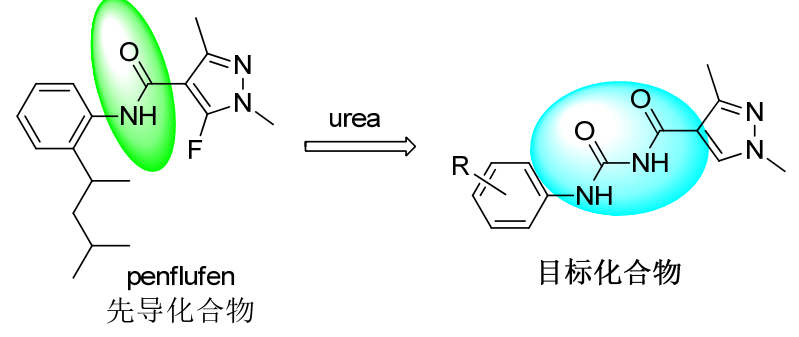

图 1 目标化合物的设计策略

Figure 1 Design strategy of title compounds<smiles>CCOC(=O)C(=COCCC(C(C)=O)C(C)=O)C(=O)OCCNN</smiles>

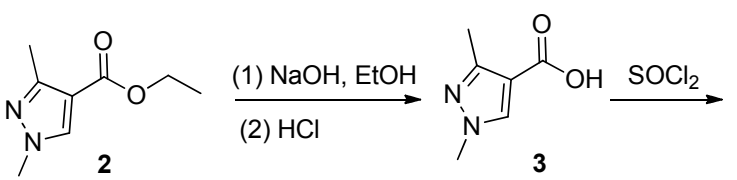<smiles>Cc1nn(C)cc1C(=O)Cl</smiles><smiles>[R]c1ccc(NC(=O)NC(=O)c2cn(C)nc2C)cc1</smiles>

图式 1 吡唑酰基脲类化合物的合成路线

Sheme 1 The synthetic route of pyrazole acylurea compounds

\section{1 结果与讨论}

\section{1 合成与波谱}

化合物的合成以乙酰乙酸乙酯与原甲酸三乙酯为 原料，经过多步反应制得关键中间体 1-甲基-3-甲基- $1 H$ -
吡唑-4-甲酰胺(5). 在合成关键中间体 $\mathbf{5}$ 的过程中，探讨 了不同反应条件对收率的影响. 最初选用四氢呋喃 (THF)为溶剂, 在冰浴下滴加氨水的 THF 溶液, 其收率 为 $54.7 \%$; 接着以丙酮作溶剂, 在冰浴下反应 $2 \mathrm{~h}$ 后倒入 水中, 无固体析出; 最后在冰浴下滴加氨水、碳酸钾的 THF 混合溶液, 室温下反应 $2 \mathrm{~h}$, 收率为 $90 \%$, 较前面两 种方法有了较大的提高. 然后与草酰氯在加热条件下形 成吡唑酰基异氰酸酯 $\mathbf{6}$, 最终将吡唑酰基异氧酸酯与取 代的苯胺反应得目标化合物 7.

以化合物 7i 为例进行说明, 化学位移 $\delta 11.26$ 和 10.18 处的单峰分别为酰基脲桥上两个 $\mathrm{N}-\mathrm{H}$ 的质子信 号峰; $\delta 8.38$ 处的单峰为吡唑环上氢的质子信号峰; $\delta$ 3.81 和 2.45 处的单峰分别为吡唑环上相连的 N-甲基和 C-甲基上氢的质子信号峰; 其余分别为苯环上氢的质子 信号峰; 同时高分辨质谱结果显示与分子量保持一致.

\section{2 杀菌活性}

番茄早疫病菌(Alternaria solani)、小麦赤霉病菌 (FusaHum graminearum)、马铃薯晚疫病菌(Phytophthora infestans)、辣椒疫霉病菌(Phytophthora capsici)、黄瓜灰 霉病菌(Botrytis cinerea)、油菜菌核病菌(Sclerotinia sclerotiorum)、水稻纹枯病菌(Thanatephorus cucumeris)、 花生褐斑病菌(Cercospora arachidicola)、苹果轮纹病菌 (Botryospuaeria berengeriana) 的杀菌活性列于表 1, 测 试浓度为 $50 \mu \mathrm{g} / \mathrm{mL}$. 由表 1 可知, 在 $50 \mathrm{mg} / \mathrm{L}$ 的浓度下, 12 个新化合物对供试菌种均表现出一定抑制活性. 但 整体来看, 在 12 种新化合物中, 化合物 $7 \mathbf{i}$ 表现出优异的 杀菌活性, 如对于油菜菌核病、水稻纹枯病、花生褐斑 病和苹果轮纹病的抑制活性与对照药氟唑菌酰胺相当, 同时对番茄早疫病、黄瓜灰霉病等也有中等的抑制活性. 此外，化合物 7a, 7e 和 7j 也同样表现出了一定的广谱杀 菌活性. 其中吡唑酰基艮类化合物对番茄早疫病、油菜

表 1 目标化合物的杀菌活性 $(50 \mathrm{mg} / \mathrm{L})$

Table 1 The fungicidal activity of title compounds $(50 \mathrm{mg} / \mathrm{L})$

\begin{tabular}{|c|c|c|c|c|c|c|c|c|c|c|}
\hline Compd. & $\mathrm{R}$ & 番茄早疫 & 小麦赤霉 & 马铃薯晚疫 & 辣椒疫霉 & 油菜菌核 & 黄瓜灰霉 & 水稻纹枯 & 花生褐斑 & 苹果轮纹 \\
\hline $7 a$ & $4-\mathrm{Et}$ & 58.1 & 51.5 & 62.2 & 61.9 & 76.9 & 61.0 & 52.3 & 5.0 & 19.2 \\
\hline $7 b$ & $2-\mathrm{NO}_{2}-4-\mathrm{Cl}$ & 32.3 & 12.1 & 13.5 & 47.6 & 38.5 & 36.6 & 11.6 & 5.0 & 42.3 \\
\hline $7 c$ & $2,5-2 \mathrm{~F}$ & 22.6 & 21.2 & 13.5 & 23.8 & 57.7 & 36.6 & 17.4 & 60.0 & 30.8 \\
\hline $7 d$ & $2-\mathrm{NO}_{2}-4-\mathrm{CH}_{3}$ & 48.4 & 51.5 & 29.7 & 26.2 & 69.2 & 48.8 & 23.3 & 5.0 & 46.2 \\
\hline $7 e$ & $2,4-2 \mathrm{~F}$ & 64.5 & 60.6 & 56.8 & 61.9 & 76.9 & 61.0 & 52.3 & 30.0 & 76.9 \\
\hline $7 f$ & $2,6-2 \mathrm{~F}$ & 32.3 & 21.2 & 21.6 & 26.2 & 38.5 & 29.3 & 11.6 & 5.0 & 38.5 \\
\hline $7 \mathrm{~g}$ & $4-\mathrm{Cl}$ & 38.7 & 21.2 & 16.2 & 23.8 & 19.2 & 39.0 & 34.9 & 5.0 & 46.2 \\
\hline $7 \mathrm{~h}$ & $4-\mathrm{Bu}$ & 41.9 & 21.2 & 10.8 & 33.3 & 57.7 & 61.0 & 44.2 & 10.0 & 38.5 \\
\hline $7 \mathbf{i}$ & $3-\mathrm{Cl}$ & 66.7 & 14.3 & 13.6 & 8.3 & 98.2 & 45.5 & 88.4 & 100.0 & 100.0 \\
\hline $7 \mathbf{j}$ & $2-\mathrm{CH}_{3}-4-\mathrm{CF}\left(\mathrm{CF}_{3}\right)_{2}$ & 66.7 & 20.0 & 22.7 & 8.3 & 85.7 & 45.5 & 86.0 & 90.0 & 36.4 \\
\hline $7 \mathbf{k}$ & $3-\mathrm{CF}_{3}$ & 33.3 & 11.4 & 9.1 & 11.1 & 67.9 & 27.3 & 46.5 & 30.0 & 50.0 \\
\hline 71 & $2-\mathrm{OCF}_{3}$ & 22.2 & 8.6 & 18.2 & 11.1 & 26.8 & 12.1 & 11.6 & 10.0 & 4.5 \\
\hline \multicolumn{2}{|c|}{ 氟唑菌酰胺 } & 88.9 & 30.3 & 29.7 & 38.1 & 96.4 & 63.6 & 88.4 & 100 & 84.6 \\
\hline
\end{tabular}


菌核病和黄瓜灰霉病具有较好的活性. 如化合物 7a, 7e, $7 \mathbf{i}$ 和 $7 \mathbf{j}$ 对番茄早疫病的抑制活性达中等( $58 \% \sim 66 \%)$, 稍逊于对照药氟唑菌酰胺 $(88.9 \%)$. 对于油菜菌核病, 大 部分化合物表现出较好的活性，如化合物 $7 \mathbf{a}(76.9 \%)$, $7 \mathbf{e}(76.9 \%), 7 \mathbf{i}(98.2 \%)$ 和 7j (85.7\%), 尤其是化合物 $7 \mathbf{i}$ 与 对照药氟唑菌酰胺相当. 对于黄瓜灰霉病, 化合物 7a $(61.0 \%), 7$ e $(61.0 \%)$ 和 $7 \mathrm{~h}(61.0 \%)$ 的抑制活性与对照药 相当, 化合物 7d (48.8\%), $7 \mathbf{i}$ (45.5\%)和 7j (45.5\%)也表现 出了中等强度的抑制活性. 对于小麦赤霉病、马铃薯晚 疫病、辣椒疫霉病、对照药氟唑菌酰胺几乎无抑制作用, 而化合物 7a 和 7e 对小麦赤霉病、马铃薯晚疫病、辣椒 疫霉病具有中等强度的抑制活性. 从表 2 可以看出化合 物 7i 和 7j 对油菜菌核病、水稻纹枯病、花生褐斑病和 苹果轮纹病具有优异的抑制活性, 7i 和 $7 \mathbf{j}$ 对油菜菌核的 $\mathrm{EC}_{50}$ 为 7.47 和 $6.71 \mu \mathrm{g} / \mathrm{mL}$, 对水稻纹枯病的 $\mathrm{EC}_{50}$ 为 9.98 和 $9.44 \mu \mathrm{g} / \mathrm{mL}$, 对花生褐斑病的 $\mathrm{EC}_{50}$ 为 7.67 和 6.45 $\mu \mathrm{g} / \mathrm{mL}$, 其中 $7 \mathbf{i}$ 对苹果轮纹病的 $\mathrm{EC}_{50}$ 为 $10.41 \mu \mathrm{g} / \mathrm{mL}$, 与 对照药氟唑菌酰胺 $\left(\mathrm{EC}_{50}=0.7287,5.38,0.060,7.93\right.$ $\mu \mathrm{g} / \mathrm{mL}$ )相比活性稍逊. 从目标化合物结构和杀菌活性的 关系可以看出, 当苯环的取代基为间位氯取代时, 其抑 制番茄早疫病、油菜菌核病、水稻纹枯病、花生褐斑病 和苹果轮纹病的活性优于其他化合物; 而当间位的取代 基电负性增大时, 其活性降低, 说明在间位的电负性增 大不利于提高活性. 当邻对位有取代基时, 化合物的活 性稍有降低. 总体来看, 苯环上的取代基的电负性和体 积大小对于化合物活性的影响较大.

表 2 目标化合物 $7 \mathbf{i}$ 和 $7 \mathbf{j}$ 对部分菌株的 $\mathrm{EC}_{50}$ 值 Table $2 \quad \mathrm{EC}_{50}$ of compounds $7 \mathbf{i}$ and $7 \mathbf{j}$

\begin{tabular}{ccccc}
\hline Compd. & 油菜菌核病 & 水稻纹枯病 & 花生褐斑病 & 苹果轮纹病 \\
\hline $7 \mathbf{i}$ & 7.48 & 9.98 & 7.67 & 10.41 \\
$\mathbf{7 j}$ & 6.71 & 9.44 & 6.45 & - \\
$\begin{array}{c}\text { 氟唑菌 } \\
\text { 酰胺 }\end{array}$ & 0.73 & 5.38 & 0.06 & 7.93 \\
\hline
\end{tabular}

\section{3 分子对接}

从 PDB 库(http://www.rcsb.org)下载琥珀酸脱氢酶 与萎锈灵的复合晶体(PDB:2FBW)的 PDB 文件. 蛋白结 构的预处理在 Discovery Studio 2.5 软件中进行. 同时对 目标化合物 7i 进行结构优化. 采用 CDOCK 模块进行分 子对接, 输出 20 个对接结果, 选取打分最高的对接构象 进行分析. 本工作采用结合萎锈灵的琥珀酸脱氢酶复合 物晶体结构作为对象进行分子对接研究 ${ }^{[19,20]}$. 如图 2 所 示, 目标化合物 $7 \mathbf{i}$ 与琥珀酸脱氢酶 $(\mathrm{SDH})$ 的相互作用图. 其中, 目标化合物 7i 嫝桥上的氨基和吡坐的酰基分别与 $\mathrm{C}$ 链上的 Ser39 残基形成氢键作用.

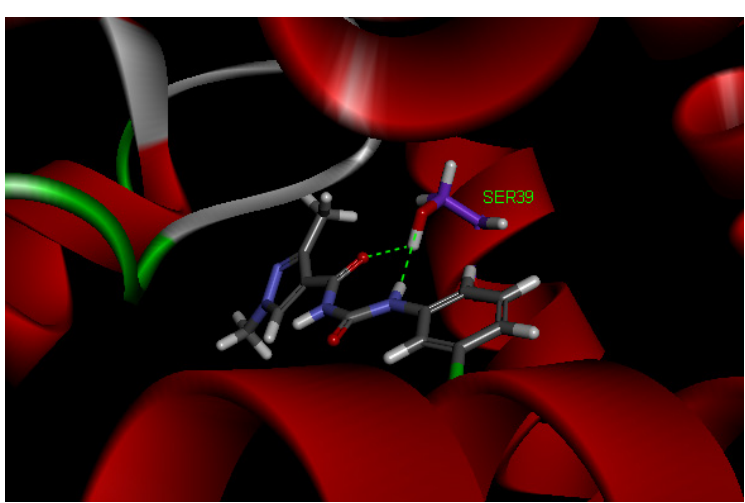

图 2 化合物 $7 \mathbf{i}$ 的分子对接图

Figure 2 Molecular docking of compound 7i

\section{2 结论}

本文以乙酰乙酸乙酯为原料, 经多步反应合成出一 系列新型吡唑酰基脲类化合物. 目标化合物经核磁共振 氢谱和高分辨质谱分析确定其结构, 并以番茄早疫病菌 (Alternaria solani)、小麦赤霉病菌(FusaHum graminearum)、马铃薯晚疫病菌(Phytophthora infestans)、辣椒 疫霉病菌(Phytophthora capsici)、黄瓜灰霉病菌(Botrytis cinerea)、油菜菌核病菌(Sclerotinia sclerotiorum)、水稻 纹枯病菌(Thanatephorus cucumeris)、花生褐斑病菌 (Cercospora arachidicola)、苹果轮纹病菌(Botryospuaeria berengeriana)分别进行了杀菌活性笁选. 结果显示化合 物 7i 对花生褐斑病菌、苹果轮纹病菌、水稻纹枯病菌、 油菜菌核病菌具有较好的杀菌活性, 可以作为先导化合 物进一步优化.

\section{3 实验部分}

\section{1 仪器与试剂}

北京泰克仪器有限公司 X-4 数字显示显微熔点仪, 温度计未校正; BRUKER $400 \mathrm{MHz}$ 或 $500 \mathrm{MHz}$ 核磁共 振仪 ( $\mathrm{CDCl}_{3}$ 为溶剂, TMS 为内标); 安捷伦 LC-QTOFMS 高分辨质谱仪. 试剂均采用分析纯试剂, 未经处理, 直接使用.

$$
\text { 关键中间体 1 4 参考文献方法 }{ }^{[21 ~ 24]} \text { 合成. }
$$

\section{2 化合物的合成}

\subsubsection{1,3-二甲基- $1 H$-吡唑-4-甲酸(3)的合成}

将乙酰乙酸乙酯 $(5.2 \mathrm{~g}, 40 \mathrm{mmol}) 、$ 原甲酸三乙酯 $(8.9 \mathrm{~g}, 60 \mathrm{mmol})$ 在乙酸酐 $(12.3 \mathrm{~g}, 0.12 \mathrm{~mol})$ 中回流反应 8 $\mathrm{h}$, 反应结束后减压蒸馏, 得粗产品 1 . 然后在冰浴下, 将中间体 1 (7.5 g, $40 \mathrm{mmol}$ )滴加到 $60 \%$ 的甲基肼 (4.6 g, $60 \mathrm{mmol})$ 与乙醇 $(20 \mathrm{~mL})$ 混合液中, $60{ }^{\circ} \mathrm{C}$ 反应 $8 \mathrm{~h}$, 反应 结束后减压蒸馏, 加入乙酸乙酯 $(20 \mathrm{~mL})$ 与饱和食盐水 萃取, 有机层用无水硫酸钠除水后悬蒸, 得 1-甲基-3-三 
氟甲基- $1 H$-吡唑-4-甲酸乙酯(2), 然后将吡唑酯 2 在氢氧 化钠的乙醇溶液中进行室温搅拌 $12 \mathrm{~h}$, 去除溶剂乙醇 后, 将固体溶解到水中, 加入盐酸, 析出白色固体 $\mathbf{3}$, 抽 滤烘干. ${ }^{1} \mathrm{H} \mathrm{NMR}\left(\mathrm{CDCl}_{3}, 500 \mathrm{MHz}\right) \delta: 7.87$ (s, 1H, pyrazole-H), 3.88 (s, 3H, N-CH 3$), 2.48$ (s, 3H, pyrazole- $\left.\mathrm{CH}_{3}\right)$.

\subsubsection{1,3-二甲基- $1 H$-吡唑-4-甲酰胺(5)的合成}

将 $14 \mathrm{~g}$ (0.1 mol) 1,3-二甲基- $1 H$-吡唑-4-甲酸(3)加 入 $250 \mathrm{~mL}$ 单口烧瓶中, 慢慢加入 $75 \mathrm{~mL} \mathrm{SOCl}_{2}$, 加热回 流 $4 \mathrm{~h}$, 薄层色谱(TLC)检测反应完全, 减压蒸馏除去 $\mathrm{SOCl}_{2}$, 得淡黄色固体 4. 在 $500 \mathrm{~mL}$ 单口烧瓶中加入 34 $\mathrm{g}$ 氨水 $(25 \%)$ 和 $15.2 \mathrm{~g} \mathrm{~K}_{2} \mathrm{CO}_{3}$, 再加入 $100 \mathrm{~mL}$ THF 稀释, 搅拌溶解后冷却至 $0{ }^{\circ} \mathrm{C}$, 冰浴下滴入上述淡黄色固体 溶于 $100 \mathrm{~mL}$ THF 的混合液, 有白色固体产生, 滴完后 室温反应 $2 \mathrm{~h}, \mathrm{TLC}$ 检测反应完全, 减压蒸馏除去 THF, 生成大量固体, 抽滤, 水洗, 干燥, 得白色固体 $511.2 \mathrm{~g}$, 熔点为 $170 \sim 171{ }^{\circ} \mathrm{C}$ (文献值 ${ }^{[25]} 172 \sim 173{ }^{\circ} \mathrm{C}$ ), 两步总 收率 $81 \% .{ }^{1} \mathrm{H} \mathrm{NMR}\left(\mathrm{CDCl}_{3}, 500 \mathrm{MHz}\right) \delta$ : $8.03(\mathrm{~s}, 1 \mathrm{H}$, pyrazole-H), $7.25\left(\mathrm{~s}, 1 \mathrm{H}, \mathrm{NH}_{2}\right), 6.82\left(\mathrm{~s}, 1 \mathrm{H}, \mathrm{NH}_{2}\right), 3.75$ (s, $\left.3 \mathrm{H}, \mathrm{N}-\mathrm{CH}_{3}\right), 2.51$ (s, 3H, pyrazole- $\left.\mathrm{CH}_{3}\right)$.

\subsection{3 目标化合物 $7 \mathbf{a} \sim 71$ 的合成}

将 $1.39 \mathrm{~g}$ (10 mmol) 1,3-二甲基- $1 H$-吡唑-4-甲酰胺 (5)溶于 $80 \mathrm{~mL} \mathrm{CH}_{2} \mathrm{Cl}_{2}$ 中, 搅拌均匀, 慢慢滴入 $1.9 \mathrm{~g} \mathrm{(15}$ $\mathrm{mmol}$ )草酰氯, 发现有气泡产生, 室温搅拌 $1 \mathrm{~h}$, 然后加 热回流 $7 \mathrm{~h}$, 反应液由乳白色变为澄清, TLC 检测反应完 全, 冷却后减压蒸馏, 残留物为淡黄色固体 $1.5 \mathrm{~g}$, 收率 为 $91 \%$, 无需纯化立即投入下一步反应. 将 $0.33 \mathrm{~g}(2$ $\mathrm{mmol}$ )吡唑酰基异氰酸酯溶于 $7 \mathrm{~mL}$ 的 $\mathrm{CH}_{2} \mathrm{Cl}_{2}$ 中, 滴入 2 $\mathrm{mmol}$ 的取代苯胺 (对于固体的取代苯胺, 将其溶于 $\mathrm{CH}_{2} \mathrm{Cl}_{2}$ 后再滴入), 加完后室温搅拌 $12 \mathrm{~h}$, 生成固体, 抽 滤, $\mathrm{CH}_{2} \mathrm{Cl}_{2}$ 洗涤, 干燥, 得系列化合物 $7 \mathbf{a} \sim 7 \mathbf{7 1}$.

$N$-(4-乙基苯基)-1,3-二甲基- $1 H$-吡唑-4-酰基脲(7a): 收率 40.2\%, 白色晶体, m.p. 235 236 ${ }^{\circ} \mathrm{C} ;{ }^{1} \mathrm{H} \mathrm{NMR}$ $\left(\mathrm{CDCl}_{3}, 400 \mathrm{MHz}\right) \delta: 10.98(\mathrm{~s}, 1 \mathrm{H}, \mathrm{NHC}=\mathrm{O}), 10.14(\mathrm{~s}$, $1 \mathrm{H}, \operatorname{ArNH}), 8.41(\mathrm{~s}, 1 \mathrm{H}$, pyrazole-H), $7.49(\mathrm{~d}, J=8.1 \mathrm{~Hz}$, 2H, ArH), 7.21 (d, $J=8.1 \mathrm{~Hz}, 2 \mathrm{H}, \mathrm{ArH}), 3.77$ (s, 3H, $\mathrm{NCH}_{3}$ ), 2.67 (q, $\left.J=7.6 \mathrm{~Hz}, 2 \mathrm{H}, \mathrm{CH}_{2}\right), 2.54$ (s, 3H, pyrazole- $\mathrm{CH}_{3}$ ), 1.25 (t, $J=7.6 \mathrm{~Hz}, 3 \mathrm{H}, \mathrm{CH}_{3}$ ); ESI-HRMS calcd for $\mathrm{C}_{15} \mathrm{H}_{19} \mathrm{~N}_{4} \mathrm{O}_{2}[\mathrm{M}+\mathrm{H}]^{+}$287.1503, found 287.1499.

$N$-(2-硝基-4-氯苯基)-1,3-二甲基- $1 H$-吡唑-4-酰基脲 (7b): 收率 47.5\%, 黄色固体, m.p. $197 \sim 199{ }^{\circ} \mathrm{C} ;{ }^{1} \mathrm{H}$ NMR $\left(\mathrm{CDCl}_{3}, 400 \mathrm{MHz}\right) \delta: 12.53(\mathrm{~s}, 1 \mathrm{H}, \mathrm{NHC}=\mathrm{O}), 8.52$ (d, $J=9.1 \mathrm{~Hz}, 1 \mathrm{H}$, ArNH) $8.31(\mathrm{~s}, 1 \mathrm{H}$, pyrazole-H), 8.18 (s, 1H, ArH), 7.93 (s, 1H, ArH), 7.57 (d, J=9.1 Hz, 1H, ArH), 3.86 (s, 3H, $\left.\mathrm{NCH}_{3}\right), 2.54$ (s, 3H, pyrazole- $\left.\mathrm{CH}_{3}\right)$; ESI-HRMS calcd for $\mathrm{C}_{13} \mathrm{H}_{13} \mathrm{ClN}_{5} \mathrm{O}_{4}[\mathrm{M}+\mathrm{H}]^{+} 338.0651$, found 338.0648 .

$N$-(2,5-二氟苯基)-1,3-二甲基- $1 H$-吡唑-4-酰基脲 (7c): 收率 54.4\%, 白色晶体, m.p. 222 $224{ }^{\circ} \mathrm{C} ;{ }^{1} \mathrm{H}$ NMR $\left(\mathrm{CDCl}_{3}, 400 \mathrm{MHz}\right) \delta: 11.46(\mathrm{~s}, 1 \mathrm{H}, \mathrm{NHC}=\mathrm{O}), 9.52$ (s, 1H, ArNH), $8.28(\mathrm{~s}, 1 \mathrm{H}$, pyrazole-H), $8.09 \sim 8.00(\mathrm{~m}$, $1 \mathrm{H}, \mathrm{ArH}), 7.17 \sim 7.11(\mathrm{~m}, 1 \mathrm{H}, \mathrm{ArH}), 6.81$ (s, 1H, ArH), $3.92\left(\mathrm{~s}, 3 \mathrm{H}, \mathrm{NCH}_{3}\right), 2.57\left(\mathrm{~s}, 3 \mathrm{H}\right.$, pyrazole- $\left.\mathrm{CH}_{3}\right)$; ESIHRMS calcd for $\mathrm{C}_{13} \mathrm{H}_{13} \mathrm{~F}_{2} \mathrm{~N}_{4} \mathrm{O}_{2}[\mathrm{M}+\mathrm{H}]^{+}$295.1001, found 295.1004 .

$\mathrm{N}$-(2-硝基-4-甲基苯基)-1,3-二甲基- $1 H$-吡唑-4-酰基 嫝 (7d): 收率 42.6\%, 黄色固体, m.p. > $300{ }^{\circ} \mathrm{C} ;{ }^{1} \mathrm{H}$ NMR $\left(\mathrm{CDCl}_{3}, 400 \mathrm{MHz}\right) \delta: 12.42(\mathrm{~s}, 1 \mathrm{H}, \mathrm{NHC}=\mathrm{O}), 8.56$ (s, 1H, ArNH), 8.38 (d, $J=8.4 \mathrm{~Hz}, 1 \mathrm{H}$, pyrazole-H), 8.01 (s, 2H, ArH), 7.46 (d, J=8.9 Hz, 1H, ArH), 3.87 (s, 3H, $\mathrm{NCH}_{3}$ ), 2.58 (s, 3H, pyrazole- $\mathrm{CH}_{3}$ ), 2.44 (s, 3H, Ar- $\mathrm{CH}_{3}$ ); ESI-HRMS calcd for $\mathrm{C}_{14} \mathrm{H}_{16} \mathrm{~N}_{5} \mathrm{O}_{4}[\mathrm{M}+\mathrm{H}]^{+}$318.1197, found 318.1197 .

$N$-(2,4-二氟苯基)-1,3-二甲基- $1 H$-吡唑-4-酰基脲 (7e): 收率 $39.1 \%$, 白色晶体, m.p. 236 $238{ }^{\circ} \mathrm{C} ;{ }^{1} \mathrm{H}$ NMR $\left(\mathrm{CDCl}_{3}, 400 \mathrm{MHz}\right) \delta: 11.48(\mathrm{~s}, 1 \mathrm{H}, \mathrm{NHC}=\mathrm{O}), 9.67$ (s, 1H, ArNH), 8.31 (s, 1H, pyrazole-H), 8.08 $7.99(\mathrm{~m}$, $1 \mathrm{H}, \mathrm{ArH}), 7.19 \sim 7.10(\mathrm{~m}, 1 \mathrm{H}, \mathrm{ArH}), 6.84 \sim 6.78(\mathrm{~m}, 1 \mathrm{H}$, ArH), 3.92 (s, 3H, $\left.\mathrm{NCH}_{3}\right), 2.56$ (s, 3H, pyrazole- $\left.\mathrm{CH}_{3}\right)$; ESI-HRMS calcd for $\mathrm{C}_{13} \mathrm{H}_{13} \mathrm{~F}_{2} \mathrm{~N}_{4} \mathrm{O}_{2}[\mathrm{M}+\mathrm{H}]^{+}$295.1001, found 295.1000 .

$N$-(2,6-二氟苯基)-1,3-二甲基- $1 H$-吡唑-4-酰基脲 (7f): 收率 47.6\%, 白色固体, m.p. $227 \sim 229{ }^{\circ} \mathrm{C} ;{ }^{1} \mathrm{H}$ $\mathrm{NMR}\left(\mathrm{CDCl}_{3}, 400 \mathrm{MHz}\right) \delta: 10.61(\mathrm{~s}, 1 \mathrm{H}, \mathrm{NHC}=\mathrm{O}), 10.54$ (s, 1H, ArNH), $8.30(\mathrm{~s}, 1 \mathrm{H}$, pyrazole-H), 7.27 (m, 1H, ArH), 7.02 (t, $J=8.0 \mathrm{~Hz}, 2 \mathrm{H}, \mathrm{ArH}), 3.68\left(\mathrm{~s}, 3 \mathrm{H}, \mathrm{NCH}_{3}\right), 2.50$ (s, $3 \mathrm{H}$, pyrazole- $\mathrm{CH}_{3}$ ); ESI-HRMS calcd for $\mathrm{C}_{13} \mathrm{H}_{13} \mathrm{~F}_{2} \mathrm{~N}_{4} \mathrm{O}_{2}$ $[\mathrm{M}+\mathrm{H}]^{+}$295.1001, found 295.0999.

$N$-(4-氯苯基)-1,3-二甲基- $1 H$-吡唑-4-酰基脲(7g): 收率 68.5\%, 白色固体, m.p. 229 $230{ }^{\circ} \mathrm{C} ;{ }^{1} \mathrm{H}$ NMR $\left(\mathrm{CDCl}_{3}, 400 \mathrm{MHz}\right) \delta: 11.11(\mathrm{~s}, 1 \mathrm{H}, \mathrm{NHC}=\mathrm{O}), 9.87(\mathrm{~s}, 1 \mathrm{H}$, ArNH), 8.29 (s, 1H, pyrazole-H), 7.54 (d, J=7.9 Hz, 2H, ArH), 7.35 (d, J=7.9 Hz, 2H, ArH), 3.82 (s, 3H, $\mathrm{NCH}_{3}$ ), 2.55(s, 3H, pyrazole- $\left.\mathrm{CH}_{3}\right) ;{ }^{13} \mathrm{C} \mathrm{NMR}\left(\mathrm{CDCl}_{3}, 125 \mathrm{MHz}\right) \delta$ : $13.51,38.62,112.18,121.35,127.13,128.76,133.93$, $136.79,150.56,151.46,164.34$; ESI-HRMS calcd for $\mathrm{C}_{18} \mathrm{H}_{16} \mathrm{~F}_{3} \mathrm{~N}_{6} \mathrm{~S}_{2}[\mathrm{M}+\mathrm{H}]^{+}$293.0800, found 293.0800.

$N$-(4-叔丁基苯基)-1,3-二甲基- $1 H$-吡唑-4-酰基脲 (7h): 收率 $17.5 \%$, 白色固体, m.p. $182 \sim 185{ }^{\circ} \mathrm{C} ;{ }^{1} \mathrm{H}$ $\operatorname{NMR}\left(\mathrm{CDCl}_{3}, 400 \mathrm{MHz}\right) \delta: 10.95(\mathrm{~s}, 1 \mathrm{H}, \mathrm{NHC}=\mathrm{O}), 10.38$ (s, 1H, ArNH), 8.49 (s, 1H, pyrazole-H), 7.49 (d, $J=8.1$ 
$\mathrm{Hz}, 2 \mathrm{H}, \mathrm{ArH}), 7.41$ (d, J=8.1 Hz, 2H, ArH), 3.77 (s, 3H, $\left.\mathrm{NCH}_{3}\right), 2.57$ (s, 3H, pyrazole- $\left.\mathrm{CH}_{3}\right), 1.34\left(\mathrm{~s}, 9 \mathrm{H}, \mathrm{C}\left(\mathrm{CH}_{3}\right)_{3}\right)$; ESI-HRMS calcd for $\mathrm{C}_{17} \mathrm{H}_{23} \mathrm{~N}_{4} \mathrm{O}_{2}[\mathrm{M}+\mathrm{H}]^{+}$315.1816, found 315.1809 .

$N$-(3-氯苯基)-1,3-二甲基- $1 H$-吡唑-4-酰基嫝(7i)：收 率 47.9\%，淡黄色固体，m.p. 201 203 ${ }^{\circ} \mathrm{C}$; ${ }^{1} \mathrm{H}$ NMR $\left(\mathrm{CDCl}_{3}, 400 \mathrm{MHz}\right) \delta: 11.26(\mathrm{~s}, 1 \mathrm{H}, \mathrm{NHC}=\mathrm{O}), 10.18(\mathrm{~s}$, 1H, ArNH), 8.38 (s, 1H, pyrazole-H), 7.76 (s, 1H, ArH), $7.32 \sim 7.27(\mathrm{~m}, 2 \mathrm{H}, \mathrm{ArH}), 7.14(\mathrm{~d}, J=7.4 \mathrm{~Hz}, 1 \mathrm{H}, \mathrm{ArH})$, $3.81\left(\mathrm{~s}, \quad 3 \mathrm{H}, \quad \mathrm{NCH}_{3}\right), 2.52\left(\mathrm{~s}, 3 \mathrm{H}\right.$, pyrazole- $\left.\mathrm{CH}_{3}\right)$; ESI-HRMS calcd for $\mathrm{C}_{13} \mathrm{H}_{14} \mathrm{ClN}_{4} \mathrm{O}_{2}[\mathrm{M}+\mathrm{H}]^{+}$293.0800, found 293.0800 .

$N$-(2-甲基-4-七氟异丙基苯基)-1,3-二甲基-1 $H$-吡唑4-酰基脲 (7j): 收率 $15.9 \%$, 粉色固体, m.p. 222 $223{ }^{\circ} \mathrm{C} ;{ }^{1} \mathrm{H}$ NMR $\left(\mathrm{CDCl}_{3}, 400 \mathrm{MHz}\right) \delta: 11.15$ (s, 1H, NH$\mathrm{C}=\mathrm{O}), 9.82$ (s, 1H, ArNH), 8.24 (s, 1H, pyrazole-H), 8.14 (d, $J=8.4 \mathrm{~Hz}, 1 \mathrm{H}, \mathrm{ArH}), 7.51 \sim 7.41(\mathrm{~m}, 2 \mathrm{H}, \operatorname{ArH}), 3.70$ $\left(\mathrm{s}, 3 \mathrm{H}, \mathrm{NCH}_{3}\right), 2.51\left(\mathrm{~s}, 3 \mathrm{H}\right.$, pyrazole- $\left.\mathrm{CH}_{3}\right), 2.45(\mathrm{~s}, 3 \mathrm{H}$, $\mathrm{Ar}-\mathrm{CH}_{3}$ ); ESI-HRMS calcd for $\mathrm{C}_{17} \mathrm{H}_{16} \mathrm{~F}_{7} \mathrm{~N}_{4} \mathrm{O}_{2}[\mathrm{M}+\mathrm{H}]^{+}$ 450.0762 , found 450.0759 .

$N$-(3-三氟甲基苯基)-1,3-二甲基- $1 H$-吡唑-4-酰基脲 (7k): 收率 30.7\%, 白色固体, m.p. $186 \sim 188{ }^{\circ} \mathrm{C} ;{ }^{1} \mathrm{H}$ $\operatorname{NMR}\left(\mathrm{CDCl}_{3}, 400 \mathrm{MHz}\right) \delta: 11.34(\mathrm{~s}, 1 \mathrm{H}, \mathrm{NHC}=\mathrm{O}), 10.02$ (s, 1H, ArNH), 8.27 (s, 1H, pyrazole-H), 7.90 (s, 1H, ArH), $7.74(\mathrm{~d}, J=8.3 \mathrm{~Hz}, 1 \mathrm{H}, \operatorname{ArH}), 7.49$ (t, $J=7.9 \mathrm{~Hz}, 1 \mathrm{H}$, ArH), 7.42 (d, $J=7.8 \mathrm{~Hz}, 1 \mathrm{H}, \mathrm{ArH}), 3.73\left(\mathrm{~s}, 3 \mathrm{H}, \mathrm{NCH}_{3}\right)$, $2.53\left(\mathrm{~s}, 3 \mathrm{H}\right.$, pyrazole- $\left.\mathrm{CH}_{3}\right)$; ESI-HRMS calcd for $\mathrm{C}_{14} \mathrm{H}_{14} \mathrm{~F}_{3} \mathrm{~N}_{4} \mathrm{O}_{2}[\mathrm{M}+\mathrm{H}]^{+}$327.1063, found 327.1067.

$N$-(2-三氟甲氧基苯基)-1,3-二甲基- $1 H$-吡唑-4-酰基 脲(7l): 收率 $29.2 \%$, 白色固体, m.p. 199 $200{ }^{\circ} \mathrm{C} ;{ }^{1} \mathrm{H}$ NMR $\left(\mathrm{CDCl}_{3}, 400 \mathrm{MHz}\right) \delta: 11.53(\mathrm{~s}, 1 \mathrm{H}, \mathrm{NHC}=\mathrm{O}), 9.70$ (s, 1H, ArNH), 8.27 (s, 1H, pyrazole-H), 8.26 (s, 1H, ArH), $7.39 \sim 7.34(\mathrm{~m}, 1 \mathrm{H}, \mathrm{ArH}), 7.31$ (d, $J=8.0 \mathrm{~Hz}, 1 \mathrm{H}, \mathrm{ArH})$, $7.19(\mathrm{t}, J=7.8 \mathrm{~Hz}, 1 \mathrm{H}, \mathrm{ArH}), 3.82\left(\mathrm{~s}, 3 \mathrm{H}, \mathrm{NCH}_{3}\right), 2.56(\mathrm{~s}$, $3 \mathrm{H}$, pyrazole- $\mathrm{CH}_{3}$ ); HRMS calcd for $\mathrm{C}_{14} \mathrm{H}_{14} \mathrm{~F}_{3} \mathrm{~N}_{4} \mathrm{O}_{3}[\mathrm{M}+$ $\mathrm{H}]^{+}$343.1013, found 343.1010 .

\section{2 .2 生物活性测试}

试验对象：番茄早疫病菌(Alternaria solani)、小麦赤 霉病菌 (FusaHum graminearum)、马铃薯晚疫病菌 (Phytophthora infestans) 、辣椒疫霉病菌(Phytophthora capsici)、黄瓜灰霉病菌(Botrytis cinerea)、油菜菌核病菌 (Sclerotinia sclerotiorum)、水稻纹枯病菌(Thanatephorus cucumeris)、花生褐斑病菌(Cercospora arachidicola)、苹 果轮纹病菌(Botryospuaeria berengeriana).

试验处理 ${ }^{[26,27]}$ : 式(I)化合物用 DMF 溶解成 $1 \% \mathrm{EC}$
备用。采用菌丝生长抑制法，评价供试化合物在 50 $\mathrm{mg} / \mathrm{L}$ 剂量下对 9 种试验靶标的室内杀菌活性, 活性测试 结果如表 1 所示.

辅助材料(Supporting Information) 化合物 $7 \mathbf{a} \sim 71$ 的 ${ }^{1} \mathrm{H}$ NMR 和 HRMS 图谱. 这些材料可以免费从本刊网站 这些材料可以免费从本刊网站上下载.

\section{Referenes}

[1] Russell, P. E. Outlooks Pest Manage. 2009, 20, 122

[2] Mu, J. X.; Shi, Y. X.; Yang, M. Y.; Sun, Z. H.; Liu, X. H.; Li, B. J.; Sun, N. B. Molecules 2016, 21, 68.

[3] Elebe, H.; Rieck, H.; Dunkel, R. WO 02008195, 2002 [Chem. Abstr. 2002, 136, 151162].

[4] Elebe, H.; Rieck, H.; Dunkel, R. WO 03010149, 2003 [Chem. Abstr. 2003, 138, 137308].

[5] Du, S.; Tian, Z.; Yang, D.; Li, X.; Li, H.; Jia, C.; Che, C.; Wang, M.; Qin, Z. Molecules 2015, 20, 8395.

[6] Chen, W.; Wei, W.; Wu, C. C.; Li, Y. X.; Li, Y. H.; Yu, S. J.; Li, Z. M. Chin. J. Org. Chem. 2015, 35, 1576 (in Chinese) (陈伟, 魏巍, 吴长春, 李玉新, 李永红, 于淑晶, 李正名, 有机 化学, 2015, 35, 1576.)

[7] Chen, Y. W.; Wan, Y. Y.; Liu, Q. X.; Liu, J. B.; Xiong, L. X.; Yu, S. J.; Li, Z. M. Chin. J. Org. Chem. 2015, 35, 882 (in Chinese) (陈有为，万莹莹，刘巧霞，刘敬波，熊丽霞，于淑晶，李正名, 有机化学, 2015, 35, 882.)

[8] Li, C. K.; Jiang, L.; Wang, Y.; Wan, F. X.; Zhang, P. Z.; Li, Y.; Cui, Z. N. Chin. J. Org. Chem. 2014, 34, 2296 (in Chinese) (李成坤，姜林，王悦，万福贤，张沛之，李映，崔紫宁，有机化 学, 2014, 34, 2296.)

[9] Liu, X. H.; Wang, Q.; Sun, Z. H.; Wedge, D. E.; Becnel, J. J.; Estep, A. S.; Tan, C. X.; Weng, J. Q. Pest Manage. Sci. 2017, 73, 953.

[10] Chen, J. N.; Wang, X. F.; Li, T.; Wu, D. W.; Fu, X. B.; Zhang, G. J.; Shen, X. C.; Wang, H. S. Eur. J. Med. Chem. 2016, 107, 12.

[11] Liu, X. H.; Zhao, W.; Shen, Z. H.; Xing, J. H.; Yuan, J.; Yang, G.; Xu, T. M.; Peng, W. L. Bioorg. Med. Chem. Lett. 2016, 26, 3626

[12] Zhao, W.; Xing, J. H.; Xu, T. M.; Peng, W. L.; Liu, X. H. Front. Chem. Sci. Eng. 2017, 11, 363.

[13] Liu, X. H.; Zhai, Z. W.; Xu, X. Y.; Yang, M. Y.; Sun, Z. H.; Weng, J. Q.; Tan, C. X.; Chen, J. Bioorg. Med. Chem. Lett. 2015, 25, 5524.

[14] Zhai, Z. W.; Shi, Y. X.; Yang, M. Y.; Zhao, W.; Sun, Z. H.;Weng, J. Q.; Tan, C. X.; Liu, X. H.; Li, B. J.; Zhang, Y. G. Lett. Drug Des. Discovery 2016, 13, 521

[15] Liu, X. H.; Tan, C. X.; Weng, J. Q. Phosphorus, Sulfur Silicon Relat. Elem. 2011, 186, 552.

[16] Zhang, L. J.; Yang, M. Y.; Sun, Z. H.; Tan, C. X.; Weng, J. Q.; Wu, H. K.; Liu, X. H. Lett. Drug Des. Discovery 2014, 11, 1107.

[17] Liu, X. H.; Fang, Y. M.; Xie, F.; Zhang, R. R.; Shen, Z. H.; Tan, C. X.; Weng, J. Q.; Xu, T. M.; Huang, H. Y. Pest Manage. Sci. 2017, DOI: $10.1002 /$ ps.4556.

[18] Yan, S. L.; Yang, M. Y.; Sun, Z. H.; Min, L. J.; Tan, C. X.; Weng, J. Q.; Wu, H. K.; Liu, X. H. Lett. Drug Des. Discovery 2014, 11, 940.

[19] Zhao, W.; Shen, Z. H.; Xing, J. H.; Yang, G.; Xu, T. M.; Peng, W. L.; Liu, X. H. Chem. Pap. 2017, 71, 921.

[20] Zhao, W.; Shen, Z. H.; Xing, J. H.; Xu, T. M.; Peng, W. L.; Liu, X. H. Chin. J. Struct. Chem. 2017, 36, 423.

[21] Zhao, W.; Shen, Z. H.; Xu, T. M.; Peng, W. L.; Liu, X.-H. J. Heterocycl. Chem. 2017, 54, 1751.

[22] Liu, X. H.; Zhao, W.; Shen, Z. H.; Xing, J. H.; Xu, T. M.; Peng, W. L. Eur. J. Med. Chem. 2017, 125, 881.

[23] Zhao, W.; Shen, Z. H.; Xu, T. M.; Peng, W. L.; Liu, X. H. Lett. Drug Des. Discovery 2017, 14, 323.

[24] Shen, Z. H.; Shi, Y. X.; Yang, M. Y.; Sun, Z. H.; Weng, J. Q.; Tan, C. X.; Liu, X. H.; Li, B. J.; Zhao, W. G. Chin. J. Struct. Chem. 
2016, 35,457

[25] Vasilevskii, S. F.; Shvartsberg, M. S. Bull. Acad. Sci. USSR, Chem. Sci. 1990, 39, 1901.

[26] Liu, X. H.; Tan, C. X.; Weng, J. Q. Phosphorus, Sulfur Silicon Relat. Elem. 2011, 186, 558
[27] Zhai, Z. W.; Wang, Q.; Shen, Z. H.; Tan, C. X.; Weng, J. Q.; Liu, X. H. Chin. J. Org. Chem. 2017, 37, 232 (in Chinese). (翟志文, 汪乔, 沈钟华, 谭成侠, 翁建全, 刘幸海, 有机化学, 2017, 37, 232.)

(Cheng, F.) 